\title{
A Proportional Integral Derivative (PID) Feedback Control without a Subsidiary Speed Loop
}

M. Aboelhassan

The aim of this investigation is to design and describe the essential features of a brushless direct-current (BLDC) motor. The static and dynamical state of the BLDC-Motor is designed and calculated.

Within this frame-work, it has been shown that while working with the P-controller in conjunction with the subsidiary speed loop and $P D$-controller (with non-zero error in a steady state) without a subsidiary speed loop, there is PID-controller without a subsidiary speed loop which has zero error in a steady state. The last part of this paper is dedicated to a simulation of the circle rounds of $P$ and PID controllers with and without a subsidiary speed loop in MATLAB-SIMULINK to decide which of these controllers is suitable, available and reliable with a BLDC-Motor and their application in cutting tool machines in general.

Keywords: PID-controller, P-controller, PD-controller, feedback control, disturbance observer.

\section{Introduction}

Brushless direct-current motors (BLDCs) are so named because they have a straight-line speed-torque curve like their mechanically commutated counterparts, permanent-magnet direct-current (PMDC) motors. In PMDC motors, the magnets are stationary and the current-carrying coils rotate. The current direction is changed using a mechanical commutation process. A brushless dc motor, on the other hand has a rotor with permanent magnets and a stator with windings (the magnets rotate and the current-carrying coils are stationary). It is essentially a dc motor turned inside out. The brushes and commutator have been eliminated and the windings are connected to the control electronics. The control electronics replace the function of the commutator and energize the proper winding. The energized stator winding leads the rotor magnet, and switches just as the rotor aligns with the stator [1].

BLDC motor control requires knowledge of the rotor position and mechanism in order to commutate the motor. To sense the rotor position, BLDC motors use Hall Effect sensors to provide absolute position sensing. This results in more wires and higher cost. BLDC motors can be designed into systems that are sensor-based or sensorless. Sensorless BLDC control eliminates the need for Hall effect sensors, using the back-EMF (electromotive force) of the motor instead to estimate the rotor position. Sensorless control is essential for low-cost variable speed applications such as fans and pumps [2].

Brushless DC (BLDC) motors are widely-used in industrial applications such as machine tool drives, computer peripherals, robotics and electric propulsion. BLDC motors have many advantages. Many of these are due to the reduced maintenance of BLDC motors (no brushes), better speed versus torque characteristics, high dynamic response, long operating life, noiseless operation, higher speed ranges, compact size, controllability, high torque to volume ratio, high efficiency and low moment of inertia.

\section{Cascaded control}

Feedback is both a mechanical, process and a signal mediated response that is looped back to control the system within itself. This loop is called the feedback loop. A control system usually has input and output to the system. When the output of the system is fed back into the system as part of its input, it is called the "feedback".

Cascade control is used to enable a process having multiple lags to be controlled with the fastest possible response to process disturbances including set point changes. Cascade control is widely used in industrial processes. Conventional cascade schemes have two distinct features with two nested

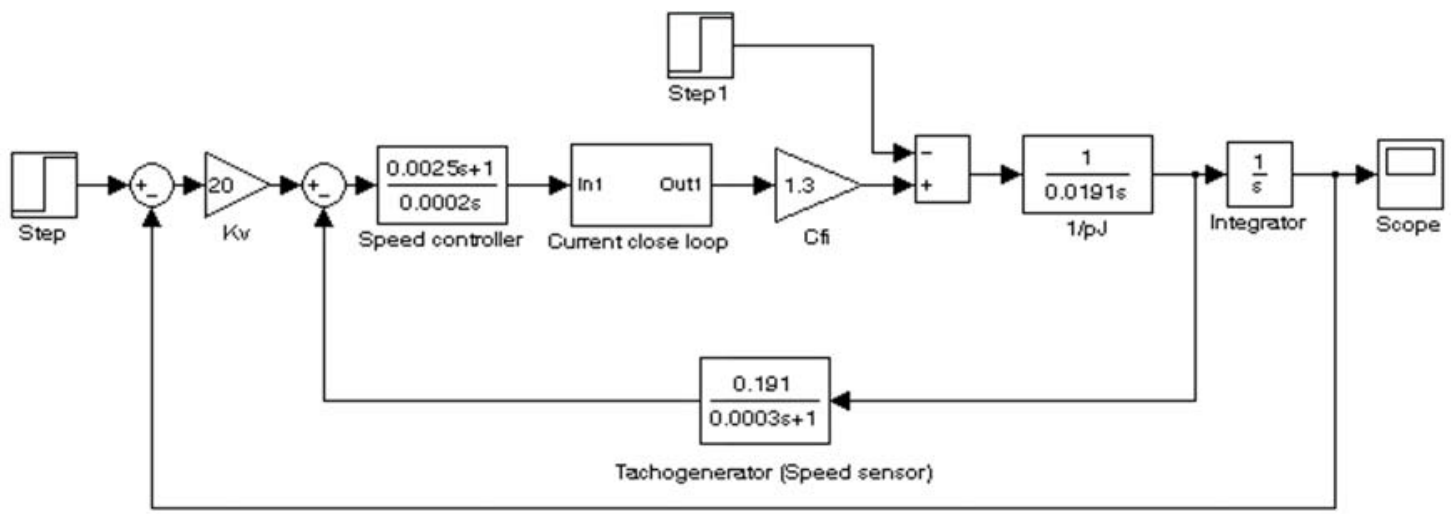

Fig. 1: Block diagram of the P-controller with the subsidiary speed loop 


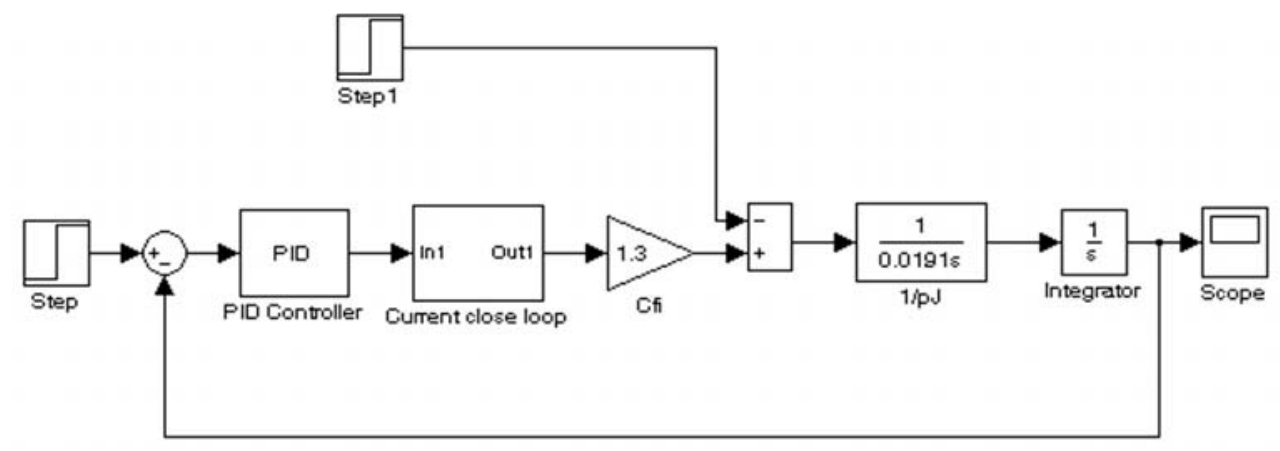

Fig. 2: Block diagram of the PID-controller without the subsidiary speed loop

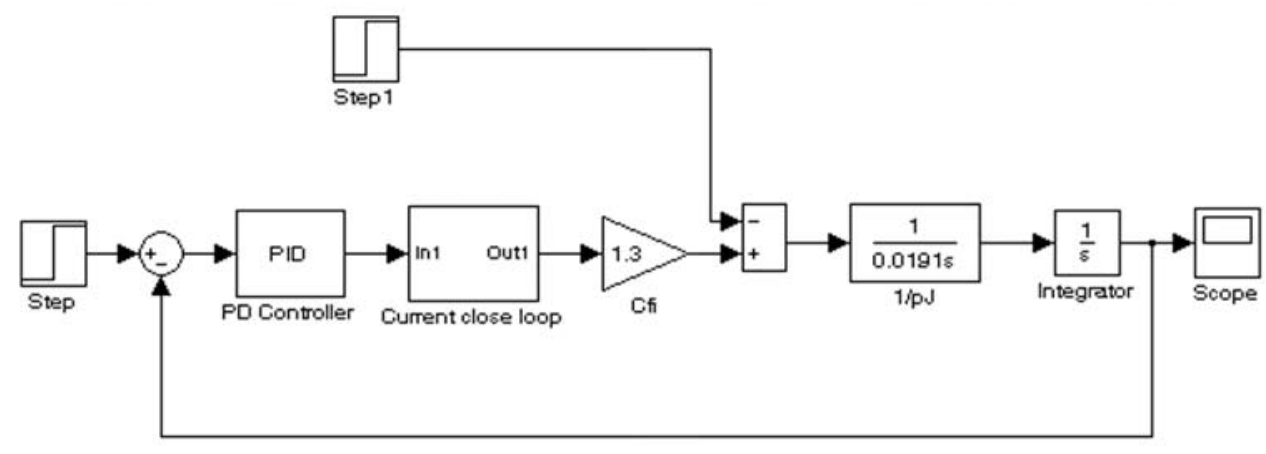

Fig. 3: Block diagram of the PD-controller without the subsidiary speed loop

feedback control loops. There is a secondary control loop located inside the primary control loop. The primary loop controller is used to calculate the setpoint for the inner (secondary) control loop. The inner loop (secondary, slave loop) in a cascade-control strategy should be tuned before the outer loop (primary, master loop). After the inner loop is tuned and closed, the outer loop should be tuned using knowledge of the dynamics of the inner loop. The most common use of a cascaded control structure is: inner current closed loop followed by speed loop and outermost position loop superimposed on the speed loop. Block diagrams of a close-loop position control system with P, PID and PD controllers with-without a subsidiary speed loop are shown in Fig. 1, Fig. 2, and Fig. 3.

\section{Comparison of P, PD, PID controllers with-without the subsidiary speed loop}

\subsection{Proportional control}

Proportional control is denoted by the P-term in the PID controller. It used when the controller action is to be proportional to the size of the process error signal $e(t)=r(t)-y_{m}(t)$. The time and Laplace domain representations for proportional control are given as [3]:

Time domain

$$
u_{C}(t)=k_{V} e(t),
$$

Laplace Domain

$$
U_{C}(s)=k_{V} E(s)
$$
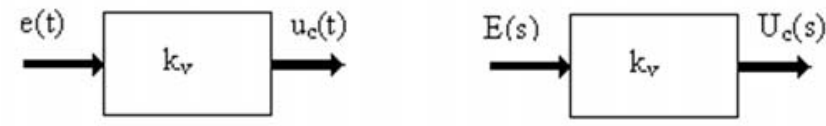

Fig. 4: Block diagrams: proportional control term

where $k_{V}$ is the proportional gain. Fig. 4 . shows the block diagrams for proportional control.

\subsection{Proportional and derivative control}

A property of derivative control that should be noted arises when the controller input error signal becomes constant but not necessarily zero, as might occur in steady state process conditions. In these circumstances, the derivative of the constant error signal is zero and the derivative controller produces no control signal. Consequently, the controller takes no action and is unable to correct for steady state offsets, for example.

To avoid the controller settling into a somnambulant state, the derivative control term is always used in combination with a proportional term. This combination is called proportional and derivative control, or PD control. The formulae for simple PD controllers are given as [3]:

Time domain

$$
u_{C}(t)=k_{V} e(t)+k_{D} \frac{\mathrm{d} e}{\mathrm{~d} t},
$$

Laplace Domain

$$
U_{C}(s)=\left[k_{V}+k_{D} s\right] E(s),
$$

where $k_{V}$ is the proportional gain and $k_{D}$ is the derivative gain. 


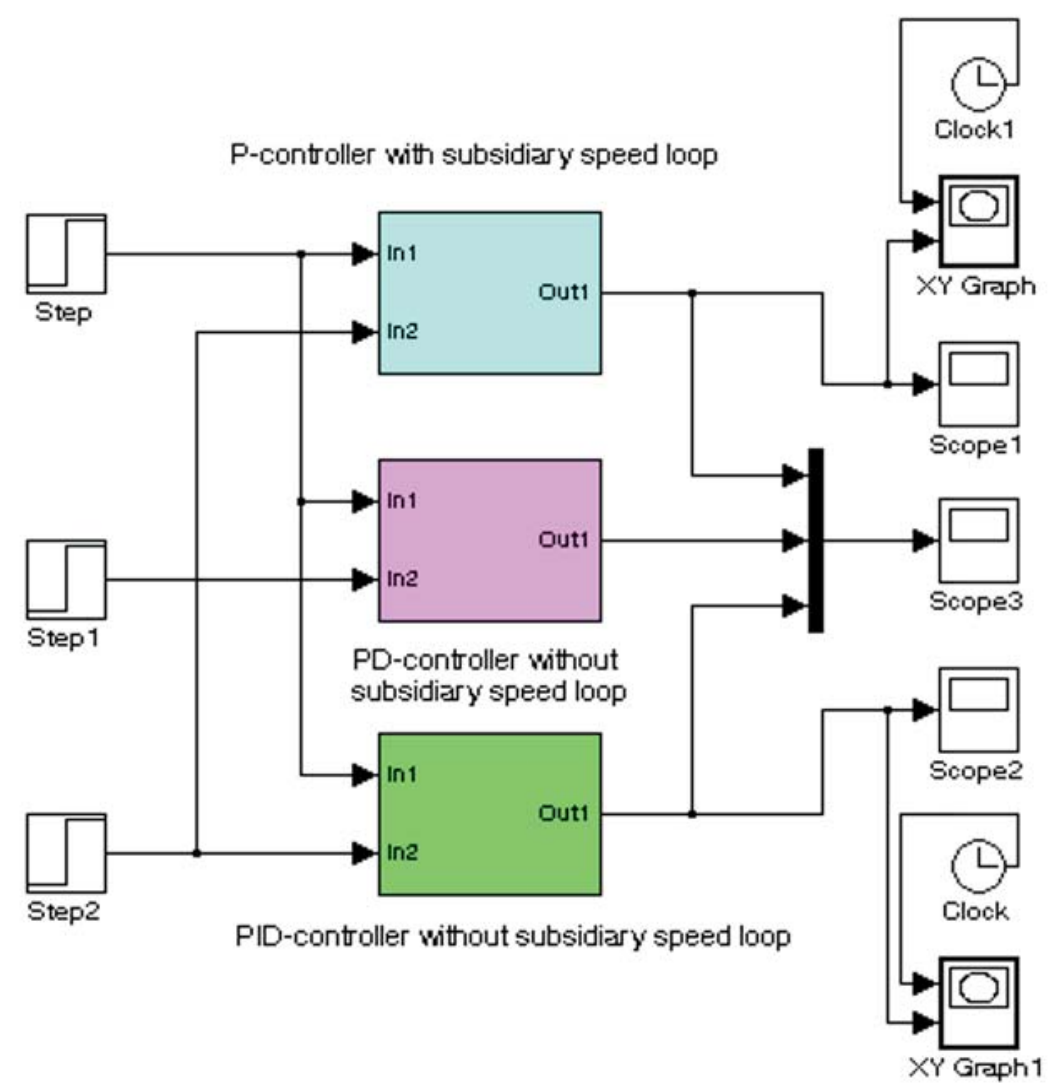

Fig. 5. Block Diagram of the P-controller, PD-controller and PID- controller with-without the subsidiary speed loop

\subsection{Parallel PID Controllers}

The family of PID controllers is constructed from various combinations of the proportional, integral and derivative terms as required to meet specific performance requirements. The formula for the basic parallel PID controller is (Transfer function PID controller formula)

$$
U_{C}(t)=\left[k_{P}+k_{I} \frac{1}{s}+k_{D} s\right] E(s) .
$$

Time-domain PID controller formula

$$
U_{C}(t)=k_{P} e(t)+k \int_{I}^{t} e(\tau) d \tau+k_{D} \frac{\mathrm{d} e}{\mathrm{~d} t} .
$$

This controller formula is often called the textbook PID controller form, because it does not incorporate any of the modifications that are usually implemented to give a working PID controller. For example, the derivative term is not usually implemented in the pure form due to adverse noise amplification properties. Other modifications that are introduced into the textbook form of PID control include those used to deal with so-called kick behavior, which arises because the textbook PID controller operates directly on the reference error signal.

This parallel or textbook formula is also known as a decoupled PID form. This is because the PID controller has three decoupled parallel paths. As can be seen from the figure, a numerical change in any individual coefficient, $k_{P}, k_{I}$ or $k_{D}$, changes only the size of the contribution in the path of the

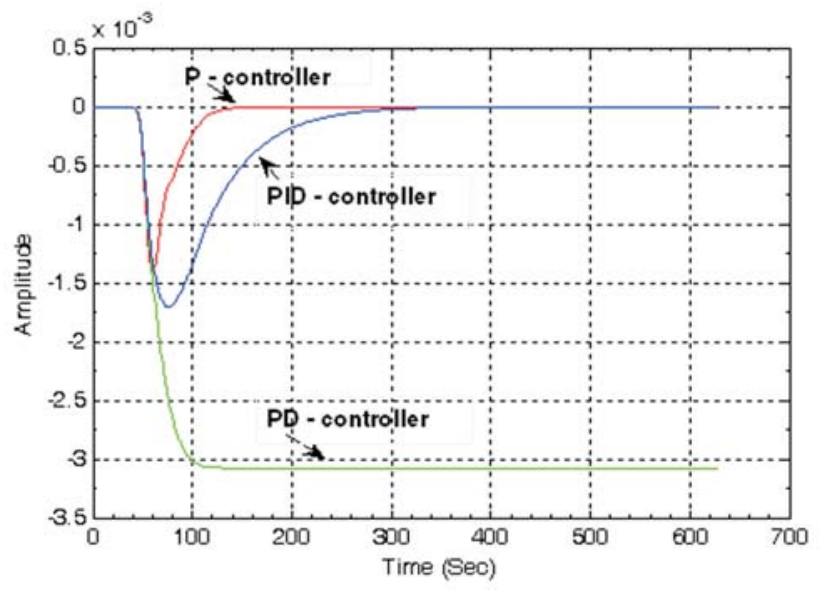

Fig. 6: Step disturbance of P, PD and PID controllers with-without subsidiary speed loop

term. For example, if the value of $k_{D}$ is changed, then only the size of the derivative action changes, and this change is decoupled and independent from the size of the proportional and integral terms. This decoupling of the three terms is a consequence of the parallel architecture of the PID controller. The block diagram of P, PD, PID controllers with-without the subsidiary speed loop is given in Fig. 5. 


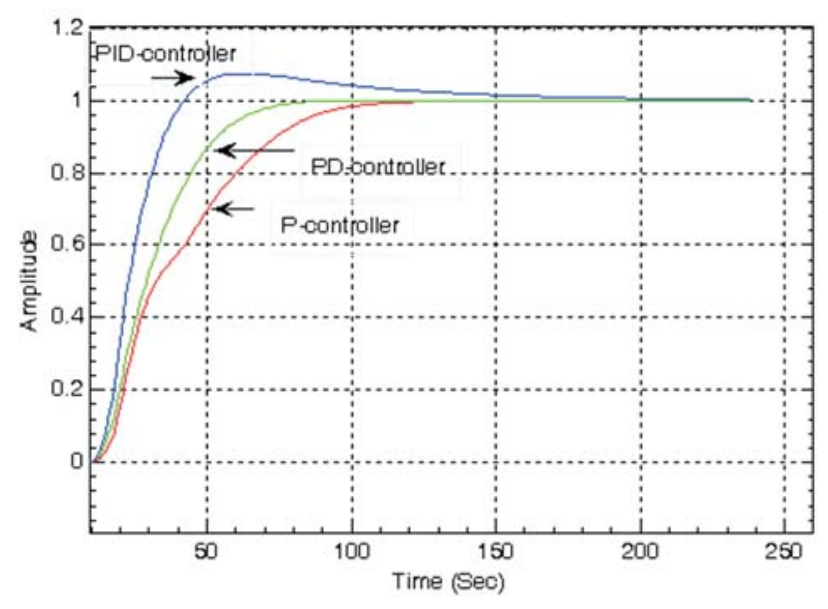

Fig. 7: Step response of P, PD a PID controllers with-without subsidiary speed loop

\section{Simulation of the circle rounds of the two regulators in MATLAB-SIMULINK}

To simulate the circle rounds we have two drives. The first moves on the $\mathrm{X}$ axis and it has a sine signal. The second is on the $\mathrm{Y}$ axis and has cosine signal. If these two drives have the same gain values, then they will have a circular movement, or else elliptical. The two drives should be the same in the same axis $\mathrm{X}$ and $\mathrm{Y}$ respectively.

A block diagram of two drives with a $\mathrm{P}_{-}$controller in conjunction with the subsidiary speed loop or a PID-controller without a subsidiary speed loop is shown in Fig. 8.

\section{Conclusion}

The simulation of two drives with the same frequency of $20 \mathrm{rad} / \mathrm{s}$ has been configured and initialized in MATLAB-SIMULINK. If these two drives have the same values of the gain $k_{V}$, they will have a circular movement, or else an elliptical movement. The increase or decrease in the frequency of the sine and cosine signal has a profound effect on the radius of the circle. Thus decreasing frequency results an increase in the radius circle, because of the frequency bandwidth of the drive. A comparison (shown in (Fig. 6:)) of P, PD, PID controllers with-without the subsidiary speed loop shows that $\mathrm{P}$ and PID controllers have zero error $(y \infty=w \infty)$ in a steady state, but the PD-controller has non-zero error.

\section{Acknowledgments}

The research described in the paper was supervised by Prof. Ing. Jiří Skalický, CSc. VUT in Brno. It has been supported by research program MSM 0021630516.

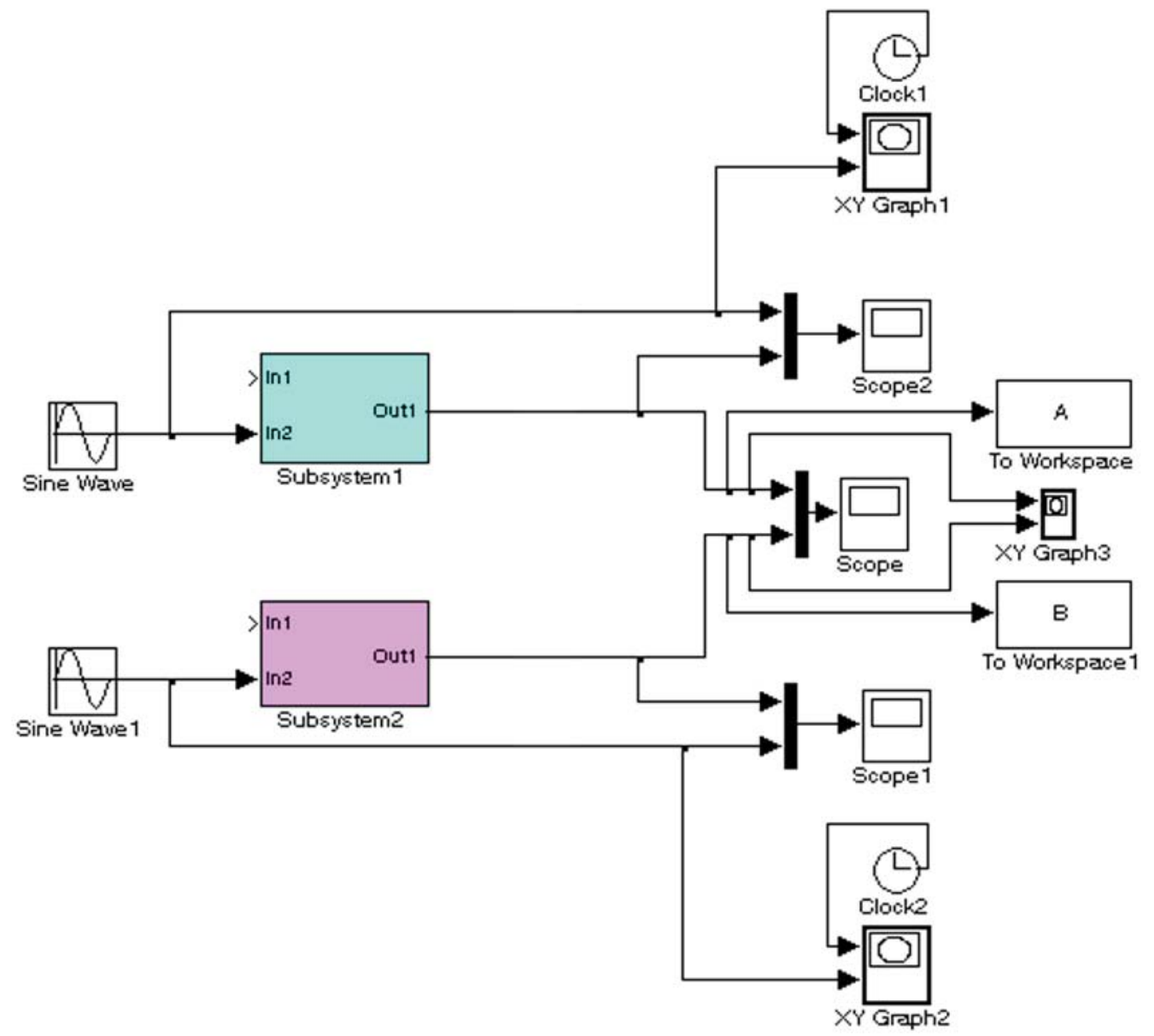

Fig. 8: Block diagram of the circle rounds for the p-controller and the State feedback controller 


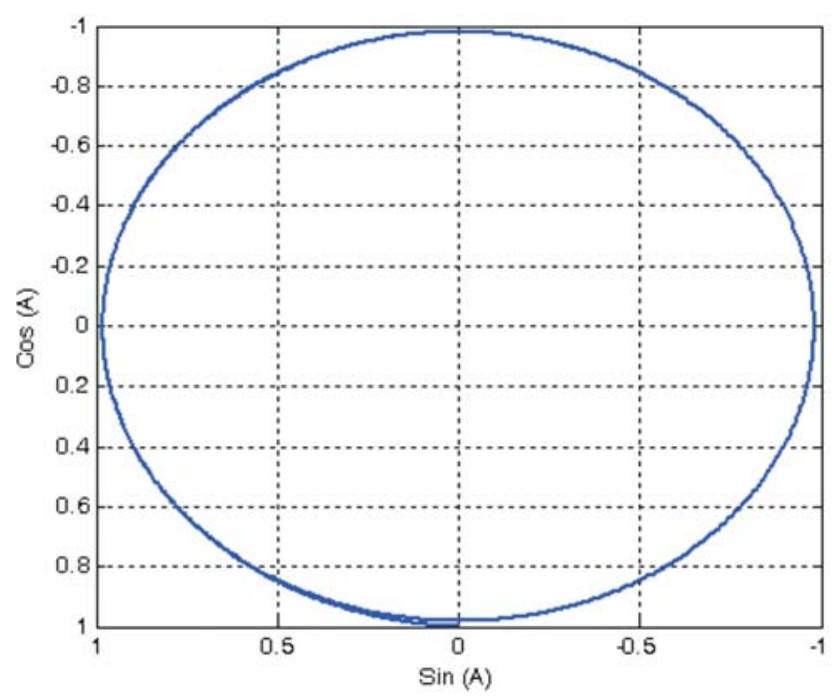

Fig. 9: Circle rounds of the $\mathrm{P}$ controller with the subsidiary speed loop and with the same gain values (freq. $\operatorname{Sin}=20 \mathrm{rad} / \mathrm{s}$ )

\section{References}

[1] Duane, D., Douglas, W.: Electronically Commutated Motors, 2001.

[2] Information on URL: http://robotika.cz/wiki/BldcMotor.

[3] Michael, A., Mohammad, H.: PID Control. New Identification and Design Methods, 2006.

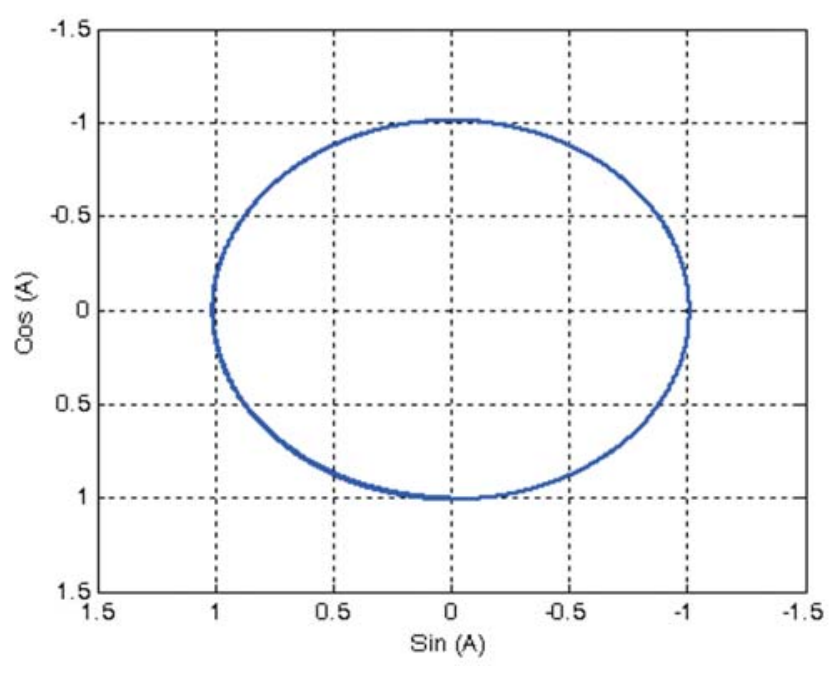

Fig. 10: Circle rounds of the PID controller without the subsidiary speed loop and with the same gain values (freq. Sin $=20 \mathrm{rad} / \mathrm{s}$ )

Mustafa Aboelhassan

e-mail: xaboel01@stud.feec.vutbr.cz

Dept. of Power Electrical and Electronic Engineering

Brno University of Technology

Technická 8

60200 Brno, Czech Republic 